\title{
DETECTION OF TNT-CONTAMINATION IN SPIKED-SOIL SAMPLES USING SPME AND GC/MS
}

\author{
E. PSILLAKIS 1 \\ G. NAXAKIS 2 and \\ N. KALOGERAKIS ${ }^{1 *}$
}

\author{
${ }^{1}$ Laboratory of Biochemical Engineering \\ \& Environmental Biotechnology \\ Department of Environmental Engineering \\ Technical University of Crete \\ Polytechnioupolis, Chania GR-731 00, Greece. \\ ${ }^{2}$ Mediterranean Agronomic Institute of Chania \\ Alsyllio Agrokipio, Chania GR-731 00, Greece \\ * to whom all correspondence should be addressed: \\ tel: $+30-821-37473$; fax: $+30+821-37474$ \\ e-mail: kalogera@mred.tuc.gr
}

Received: 22/11/99

Accepted: 07/12/00

\section{ABSTRACT}

Soil-contamination from toxic explosive residues at military bases throughout the world, is a major environmental concern. A strong candidate for TNT soil-contamination within Greece, is the military base/airport of Maleme in Crete. While soil sampling was in progress new routes of trace analysis for explosives in soil were explored. Solid-phase micro-extraction (SPME) was used to selectively preconcentrate analytes prior to GC/MS. In the preliminary experiments (effectuated on water-spiked samples) optimization of the SPME technique was achieved by controlling parameters such as sampling method, salt content and sample agitation. As immersion of the SPME fiber was found to be more efficient for extracting most analytes of interest, a pretreatment step was introduced for the soil samples which simply converted them to water samples. The developed SPME protocol was able to screen explosives in spiked-soils, in concentrations well below the certified reporting and detection limits.

KEYWORDS: explosive, GC/MS, soil contamination, SPME

\section{INTRODUCTION}

The release in nature of explosives caused by prolonged manufacturing, storage and testing of explosive weaponry is a serious environmental concern due to the toxicity of these compounds (Walsh, 1990; Jenkins and Walsh, 1989). Site investigations in US and elsewhere have shown that 2,4,6-trinintrotoluene (TNT) is the least mobile and most frequently occurring contaminating compound in the soil. High levels of TNT- contamination are of major concern since TNT and most of its biodegradation and photolytical by-products are mutagenic and carcinogenic or otherwise toxic to aquatic and terrestrial life (Reddy et al., 1997; Berthe-Corti et al., 1998). There are many contaminated sites in North America and Europe. The military base/airport of Maleme appears to be a strong candidate for explosive soil-contamination within Greece. Due to its strategic location it was extensively bom- 
barded during the World War II and up until today NATO and the Greek Air Force is using this area for shooting practice. Careful sampling design of this area revealed that in order to investigate the extent of soil-contamination from explosive residues, at least 500 samples needed to be collected and submitted to expensive and timeconsuming laboratory analyses. While sampling was still in progress, the need to develop new costand time-effective routes of analysis for the trace detection of explosives in soil became of paramount importance.

To detect explosives and their metabolites in different environmental matrices (soil or water), the U.S. Environmental Protection Agency (U.S. EPA) suggests a three-step process (U.S. EPA Method 8330, 1994). The first step involves extraction of target compounds using salting-out or solid-phase extraction for water samples or solvent extraction followed by filtration for soil samples. In the next two steps, separation and detection of the target compounds is effectuated using high performance liquid chromatography with an ultraviolet detector (HPLC-UV). Recently the introduction of gas chromatography (GC) in the trace analysis of explosives has provided another option (Hable et al., 1991; Walsh and Raney, 1998, Barshick and Griest, 1998). As these reports concerned only water samples, our focus was to develop a new alternative analytical technique for soil samples based on GC. To our knowledge, gas chromatography has not been very successful for such samples (Darrach et al., 1998), as it demands preconcentration of the acetonitrile extracts by solvent evaporation (Jenkins and Walsh, 1987). During this evaporation step, potential loss and/or deterioration of target analytes has been reported (Darrach et al., 1998). In the work presented here, extraction from soil matrices was successfully performed using the relatively new Solid-Phase Microextraction (SPME) technique, whereby the problems of solvent evaporation are avoided.

SPME is a powerful alternative to traditional techniques for the extraction of volatile or semivolatile organic compounds (Belardi and Pawliszyn, 1989; Zhang et al., 1994). It is a fast, simple, precise and sensitive technique that requires no solvent (Arthur et al., 1992a). For this technique, a thin fused silica fiber coated with a stationary phase is exposed to the target compounds in the sample, either by direct immersion or to the headspace above an aqueous or solid sample. Analytes sorb to the stationary phase until equilibrium is achieved. The fiber is then transferred directly to a heated injection port of a gas chromatograph for subsequent thermal desorption and analysis. First developed to analyze volatile chlorinated organics, polychlorinated biphenyls (PCBs), and BTEX (benzene, toluene, ethylbenzene, xylene) in water, SPME has been successfully used for a wide variety of applications, the majority of which involves the analysis of water samples (Eisert and Levsen, 1996). Headspace SPME introduced the analysis of soil samples contaminated with volatile and semivolatile organics. Quantitative analysis of analytes in soil samples using immersion SPME has not been frequently reported, due to interference of the SPME fiber with the complex soil matrix (Sarrion et al., 1998).

Although, SPME can be easily coupled to GC, SPME-HPLC coupling is more complex as it needs a special interface to desorb the analytes. This interface insures analyte desorption by adding an organic solvent to the fiber. Thus, analytes are introduced into the HPLC column by the mobile phase. As organic solvents can damage the fiber and lead to irreproducible results the SPME-GC coupling was preferred. In this work, SPME-GC/MS was applied for the trace analysis of soil samples spiked with explosives and their metabolites. Initial experiments were effectuated in water spiked samples in order to optimize extraction of the analytes by controlling several parameters. Different sampling methods such as headspace and immersion SPME were investigated as well as the effect of sample agitation and salt content. As headspace SPME did not efficiently extract all analytes of interest, immersion SPME was preferred. In order to avoid contact of the SPME fiber with the soil particles, we introduced a pretreatment step where soil samples were converted into water samples. These preliminary experiments revealed that the combination SPME-GC/MS is an excellent screening method for the trace analysis of explosive residues in soil detecting contaminants in levels lower than the ones reported in U.S. EPA Method 8330 (1994). The developed protocol reduces the cost of analysis per sample and limits the production of hazardous laboratory waste due to the traditional solvent extraction. 


\section{MATERIALS AND METHODS Chemicals}

Acetonitrile (HPLC grade) was obtained from Merck KGaA. Analytical standards of all fourteen target analytes suggested by the U.S. EPA Method 8330, were obtained from Supelco (Sigma-Aldrich Chemie $\mathrm{GmbH}$ ), in the form of two separate $1 \mathrm{ml}$ acetonitrile solutions (MixA and MixB), where MixA contained 2-amino-4,6dinitrotoluene (2-ADNT), 1,3-dinitrobenzene (1,3-DNB), 2,4-dinitrotoluene (2,4-DNT), HMX, nitrobenzene (NB), RDX, 1,3,5-trinitrobenzene (1,3,5-TNB) 2,4,6-trinitrotoluene (TNT), and MixB contained 4-amino-4,6-dinitrotoluene (4ADNT), 2,6-dinitrotoluene (2,6-DNT), 2-nitrotoluene (2-NT), 3-nitrotoluene (3-NT), 4-nitrotoluene (4-NT) and tetryl, each at $100 \mu \mathrm{g} \mathrm{ml}^{-1}$. 2,3dinitrotoluene (2,3-DNT) was used as an internal standard and was purchased from Riedel-de Haën Laborchemikalien GmbH \& Co. KG (Sigma-Aldrich Chemie $\mathrm{GmbH}$ ). Water used for preparing the solutions was type I reagent grade water from a Milli- $Q^{\circledR}$ reagent water system (Millipore Corp.). An acetonitrile solution of 2,3dinitrotoluene (2,3-DNT) (Riedel-de Haën Laborchemikalien $\mathrm{GmbH} \& \mathrm{Co} . \mathrm{KG}$ ) was used as internal standard.

\section{Preparation of calibration standards}

Working standards were prepared each day covering the range of interest. With the use of Microliter syringes (Hamilton series 701), the exact quantity of the mixed analytes was transferred to a 10-ml volumetric flask and diluted to volume with salt water $(27 \% \mathrm{w} / \mathrm{v} \mathrm{NaCl})$.

\section{Preparation of spiked-soil samples}

Soil collected from the Maleme military base/airport outside the shooting range (hand-digging in 15-cm-depth intervals), was air-dried to a constant weight and ground to pass through a $2 \mathrm{~mm}$ mesh for soils. A series of $2.00 \mathrm{~g}$ of subsamples were placed in 10-ml vials and spiked with the two multicomponent standard solutions, yielding target concentrations of $0.40,0.20$ and $0.10 \mathrm{mg} \mathrm{kg}^{-1}$ dry-soil, respectively. The samples were then allowed to stand for at least one hour, as stated in previous reports (Jenkins and Walsh, 1987). Aliquots of $8 \mathrm{ml}$ of reagent grade water $(27 \% \mathrm{w} / \mathrm{v}$ $\mathrm{NaCl})$ were added and the spiked samples were placed in an ultra-sonic bath for $2 \mathrm{~h}$. After sonica- tion, the soil-slurry samples were allowed to stand in the dark overnight. $5 \mathrm{ml}$ of supernatant were carefully removed and placed in 10-ml vials. Each vial was Teflon-capped just after adding a portion of the internal standard solution.

\section{Sample Extraction}

Solid-Phase Microextraction was performed using a manual $65 \mu \mathrm{m}$ polydimethylsiloxane /divinylbenzene (PDMS/DVB) SPME fiber and fiber holder assembly (Supelco, Sigma-Aldrich Chemie $\mathrm{GmbH}$ ). $5 \mathrm{ml}$ sample volumes were extracted by immersing the fiber in a sample that was (unless otherwise stated) stirred rapidly and consistently with a magnetic stir bar. The SPME fiber holder assembly was clamped above and resting on the vial cap. After $30 \mathrm{~min}$, the fiber was retracted into the septum-piercing needle and transferred immediately to the heated $\left(250{ }^{\circ} \mathrm{C}\right)$ injection port of the GC. The SPME fiber was then extended into the splitless injector for $5 \mathrm{~min}$. Analytes were completely desorbed after that period and no carry-over on second desorptions was found.

\section{GC/MS analysis}

The GC parameters were configured according to previous reports. All analyses were carried-out on a Hewlett-Packard 5890 Series 2 GC system coupled to a mass spectrometer VG TRIO 2000. Positive electron impact at $70 \mathrm{eV}$ was used. The ion source was maintained at $200{ }^{\circ} \mathrm{C}$. Data was acquired in the full-scan detection mode from 45 to $350 \mathrm{amu}$ at rate of $1 \mathrm{scan} \mathrm{sec}^{-1}$. A solvent delay time of 4 min was used. Sample introduction was performed using a standard split/splitless-type injector in the splitless injection mode. Splitless time was $5 \mathrm{~min}$ for SPME fiber desorptions. The injection port temperature was maintained at $250{ }^{\circ} \mathrm{C}$. Separation was performed on a $30 \mathrm{~m} \times$ $0.25 \mathrm{~mm} \times 0.25 \mu \mathrm{m}$ SPB-1701 capillary column (Supelco, Sigma-Aldrich Chemie $\mathrm{GmbH}$ ). The head-pressure was set at $60 \mathrm{kPa}$. The column oven was initially held at $100{ }^{\circ} \mathrm{C}$ for $2 \mathrm{~min}$, programmed to $200{ }^{\circ} \mathrm{C}$ at a rate of $10{ }^{\circ} \mathrm{C} \mathrm{min}^{-1}$, then to $250{ }^{\circ} \mathrm{C}$ at $20{ }^{\circ} \mathrm{C} \mathrm{min}-1$ (hold for $5 \mathrm{~min}$ ). Helium was used as a carrier gas at a $0.9 \mathrm{ml} \mathrm{min}^{-1}$ flow-rate (set at $\left.100{ }^{\circ} \mathrm{C}\right)$. 


\section{RESULTS AND DISCUSSION SPME Extraction}

There are several parameters that need to be controlled in order to ensure optimum performance of the SPME technique: fiber polarity, fiber coating thickness, sampling method (immersion versus headspace), sample medium ( $\mathrm{pH}$ and ionic strength), and rate of sample agitation. Based on previous studies the $65 \mu \mathrm{m}$ polydimethylsiloxane /divinylbenzene (PDMS/DVB) fiber was chosen for the present study, as it exhibits high selectivity for most of the target analytes present in water samples (Barshick and Griest, 1998). The remaining parameters were taken into consideration in the development of the analytical method as discussed bellow.

The choice of the sampling method (Figure 1) is quite critical. Environmental matrices such as soil are fairly complex and direct immersion sampling could damage or foul the SPME fiber. A literature report using microscopy techniques revealed that the extraction capacity of the fiber declines during such measurements, not because of the irreversible destruction of the fiber, but because of the deposition of small soil particles on the fiber (Tugulea et al., 1998). Although the authors suggested a two-step cleaning (chemical followed by mechanical), the fiber's life could be extended

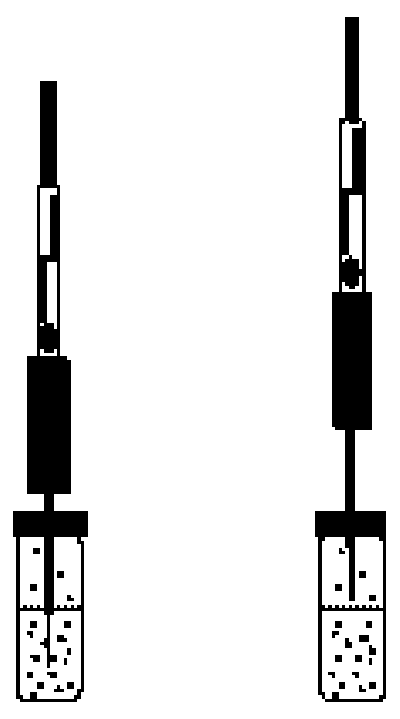

Figure 1. Immersion and headspace SPME sampling methods. for a further fifteen samplings, making SPME a rather time-consuming and expensive technique. On the other hand in headspace sampling, direct contact between soil and fiber is impossible, making it ideal for soil matrices. Headspace sampling relies on the equilibrium partitioning of the analyte among the matrix, headspace and the SPME phase, and it works well for analytes exhibiting vapor pressure (Zhang and Pawliszyn, 1993).

Subsequent comparison of the two sampling methods was effectuated on $5 \mathrm{ml}$ samples containing $500 \mu \mathrm{g} \mathrm{l}^{-1}$ of each target analyte, in reagent grade water. During these experiments proper sample agitation was applied in order to enhance extraction efficiency and reduce equilibration times by increasing the rates of diffusion.

In the headspace experiments, sonication of the sample was applied. The sonic bath was kept all the time in a constant mildly elevated temperature $\left(40{ }^{\circ} \mathrm{C}\right)$, promoting thus vaporization of the analytes. Higher temperatures were avoided for two reasons: they could result in decomposition of the target analytes as well as decrease the analyte sorption into the fiber, as sorption is an exothermic process (Frombert et al., 1996). For immersion sampling, mechanical stirring at room temperature was preferred. Although sonication proved to promote analyte sorption, we found that in immersion sampling the fiber deteriorates and results in fluctuating temperatures, and hence, decreased precision.

The results of these experiments depicted in Figure 2 show clearly that although the headspace works relatively well for analytes of lower molecular weight (NB and NTs), extraction of the larger molecules (DNB and DNTs) is not complete, or even not possible (TNT, TNB and ADNTs). Even when the sampling time, during which the fiber is exposed to the solution and adsorbs the target analytes, is prolonged, the results remain the same. This was expected considering the fact that with headspace sampling, equilibration is faster because the analytes do not have to penetrate a static layer of water molecules surrounding the fiber as with immersion (Walsh et al., 1996). Addition of salt to the sample prior to extraction can increase the ionic strength of the solution and affect the solubility of the analytes. Depending on the analyte, this can either enhance or limit extraction. Likewise, changing the $\mathrm{pH}$ can minimize solubility where acidic or basic compounds 


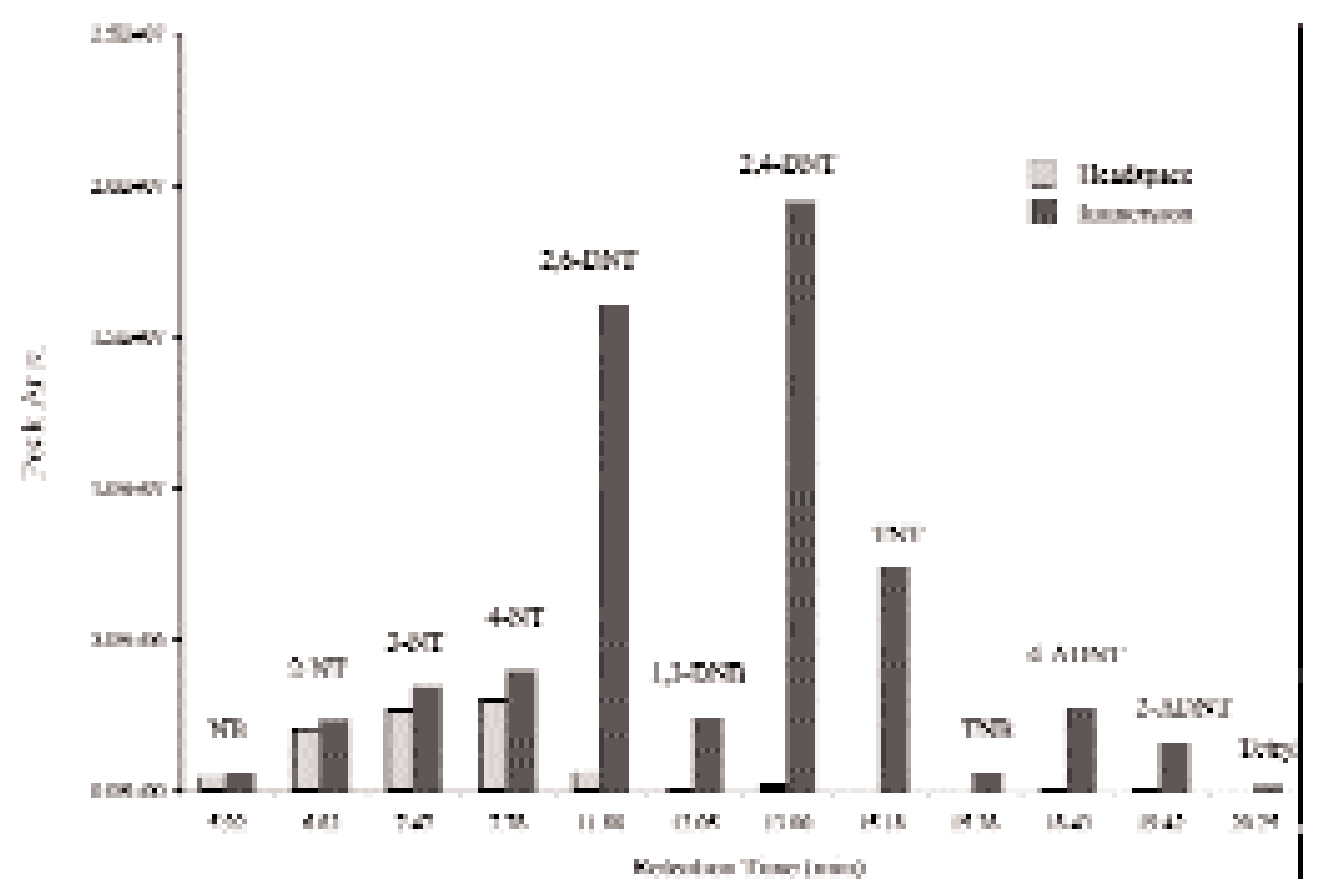

Figure 2. Relative extraction efficiencies for a $30 \mathrm{~min}$ sampling time on $5 \mathrm{ml}$ water samples, spiked with $500 \mu \mathrm{g} \mathrm{l}^{-1}$ of each target analyte, using headspace and immersion SPME sampling methods

are less soluble at low and high $\mathrm{pH}$, respectively. Recent studies revealed that sample $\mathrm{pH}$ in the range of 4-10 does not have a significant impact on extraction efficiency (Arthur et al., 1992a; 1992b). However exposure to more acidic or basic samples can cause deterioration of the fiber. Of the analytes used in this study, only the monoamino metabolites of TNT would be affected by these factors.

The extraction experiments using both sampling methods were repeated, but this time salt was added $(27 \% \mathrm{w} / \mathrm{v} \mathrm{NaCl})$ and the $\mathrm{pH}$ was found to be 8.6. For this method, at a sample $\mathrm{pH}$ of 8.6 and assuming $\mathrm{pKa}$ has a value in the range 1 to 2.5 for the nitroaniline compounds, the monoamino metabolites should be uncharged and already in a form that would be most efficiently extracted. The addition of salt, enhanced greatly extraction when immersion sampling was used, however it did not result in any significant change when the headspace sampling method was used.

Overall, immersion of the SPME fiber into the sample could not be avoided, if detection of all the analytes of interest is desired. In order to avoid soil-fiber contact, the soil particles had to be eliminated from the solution prior to extraction. This was possible by leaving the soil-slurry solution to settle overnight and use the supernatant for SPME analysis. Centrifugation can also be used to reduce the settling time. The sample conditions used for all subsequent measurements were as described in the experimental section (30 min immersion sampling, consistent magnetic stirring, $27 \% \mathrm{w} / \mathrm{v} \mathrm{NaCl}$ ).

\section{GC/MS Analysis}

As already mentioned, analyzing organic chemical residues in soil is a three-step process. Once extraction is accomplished, further determination of explosives in the extracts requires a separation step since multiple analytes are always present. Environmental analysis of explosives has been dominated so far by HPLC protocols (Jenkins and Walsh, 1989). As some of the target compounds (e.g. HMX, RDX) have high melting points, low vapor pressures, and thermal lability (Walsh and Raney, 1998), HPLC seems ideal due to the fact that analytes move through the column at ambient temperature in solution. Large sample volumes can be used, lowering the detection lim- 


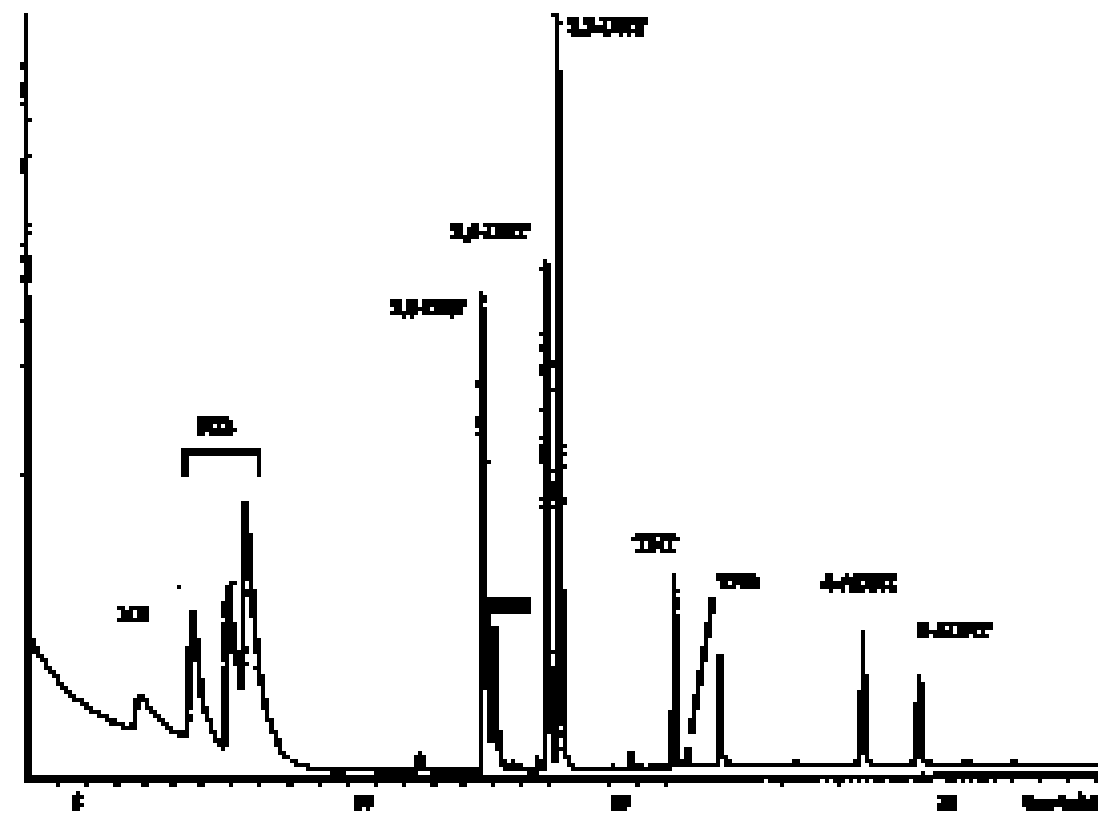

Figure 3. Gas chromatograph obtained for a $30 \mathrm{~min}$ immersion sampling on a $5 \mathrm{ml}$ water sample containing $27 \%$ of $\mathrm{NaCl}$, spiked with $50 \mu \mathrm{g} \mathrm{l}-1$ of each target analyte.

its and eliminating the need of solvent preconcentration prior to analysis. An alternative approach to HPLC relies on GC, where analytes move through the column on an elevated temperature in the gas phase and its generally agreed that it works best for the relatively volatile that are not thermally labile. Detecting unexploded ordnance with GC is analytically difficult and would lead one to believe that GC analysis is impractical. However, recent reports reveal that quantitative GC analysis of explosive contaminated water-samples is possible when deactivated injection-port liners, high injection-port temperatures and short, wide-bore capillary columns are used (Hable et al., 1991; Walsh and Raney, 1998). As pointed in the introduction, the work presented here focuses on the development of a new extraction technique for soil-contaminated samples. In these studies, optimization of the GC analysis was not our first priority. The main task was to establish whether SPME is a feasible protocol for soil samples and subsequently examine whether the combination SPME-GC/MS can lead to a reliable quantitative method for all target analytes.

As expected from previous literature reports, the use of a $30 \mathrm{~m}$ capillary column for conducting the present chromatographic separations, lead to analyte loss of thermally labile analytes. Indeed, elution of HMX and RDX was not observed and this is the reason why measurements for these compounds are not included in this paper. The absence of an inlet liner specific for SPME/GC analyses, is responsible for the peak tailing obtained for the first four elutants which is more pronounced for NB (Figure 3). Finally, the present chromatographic conditions are responsible for the small peaks obtained for TNT, TNB and tetryl. The average retention times for eluting analytes are presented in Table 1 .

\section{Detection Limits and Calibration}

Method detection limits (MDL) for SPME immersion sampling were determined using the chromatographic conditions described in the experimental section. Based on published guidelines, limit of detection was based on a confidence of $3 \sigma$ and limit of quantification on a confidence of $10 \sigma$ (U.S. EPA Method 8000B, 1996). Detection limits for most target analytes were found in the low parts per billion (ppb) range 
Table 1. Average Retention Times (ART), Method Detection Limits (MDL) and Limits of Quantification (LOQ) for eluting analytes (after SPME immersion sampling on $5 \mathrm{ml}$ water samples containing 27\% $\mathrm{w} / \mathrm{v} \mathrm{NaCl}$ and the target analytes).

\begin{tabular}{rcccrcrc}
\hline Analyte & $\begin{array}{c}\text { ART } \\
(\mathrm{min})\end{array}$ & $\begin{array}{c}\text { MDL } \\
\left(\mu \mathrm{g} \mathrm{l}^{-1}\right)\end{array}$ & $\begin{array}{c}\text { LOQ } \\
\left(\mu \mathrm{g} \mathrm{l}^{-1}\right)\end{array}$ & Analyte & $\begin{array}{c}\text { ART } \\
(\mathrm{min})\end{array}$ & $\begin{array}{c}\text { MDL } \\
\left(\mu \mathrm{g} \mathrm{l}^{-1}\right)\end{array}$ & $\begin{array}{c}\text { LOQ } \\
\left(\mu \mathrm{g} \mathrm{l}^{-1}\right)\end{array}$ \\
\hline NB & 5.88 & 10 & - & $2,4-\mathrm{DNT}$ & 12.97 & 2.5 & 5 \\
2-NT & 6.82 & 2.5 & 5 & TNT & 15.17 & 15 & - \\
3-NT & 7.44 & 2.5 & 5 & TNB & 15.40 & 25 & \\
4-NT & 7.78 & 2.5 & 5 & 2-ADNT & 18.43 & 5 & 10 \\
2,6-DNT & 11.86 & 2.5 & 5 & 4-ADNT & 19.41 & 5 & 10 \\
1,3-DNB & 12.04 & 2.5 & 5 & Tetryl & 20.26 & 125 & - \\
\hline
\end{tabular}

(Table 1). Tetryl showed the highest detection limit at $125 \mu \mathrm{g} \mathrm{l}^{-1}$. The present chromatographic conditions as well as the instability of tetryl in the commercially available acetonitrile multicomponent solutions were encountered for this observation (Hable et al., 1991; Jenkins and Walsh, 1989). The broad and asymmetric peak corresponding to NB resulted in a detection limit of the order of $10 \mu \mathrm{g} \mathrm{l}^{-1}$. Overall, NB, TNT, TNB and tetryl were not included in the recovery experiments, as the instruments response was small when compared to the rest of the analytes (higher detection limits). Their limits of quantification were not calculated.

Prior to any quantitative measurements, the relationship between the response of an instrument and the amount or concentration of an analyte introduced into the instrument, must be established (calibration). The SW-846 criterion of linearity of this relationship is that the Relative Standard Deviation (RSD) of the Mean Response Factor (MRF) be less or equal to $15 \%$ for standards containing the target analytes at a minimum of five different concentrations covering the range of interest (U.S. EPA Method 8000B, 1996).

The calibration data (summarized in Table 2) for all quantifiable analytes, showed linear models, relating responses to concentrations over the entire range of concentration tested. The Mean Response Factors (MRFs) was used for all further calculations.

Table 2. Response factors and Relative Standard Deviations for all analytes with a calculated Limit of Quantification

\begin{tabular}{rcccccccc}
\hline \multicolumn{8}{c}{ Response Factors $^{\mathbf{a}}$ in the range 100-5 } & ì $\mathbf{l}^{-\mathbf{1}}$ \\
\hline & $\mathbf{1 0 0}$ & $\mathbf{7 5}$ & $\mathbf{5 0}$ & $\mathbf{2 5}$ & $\mathbf{1 0}$ & $\mathbf{5}$ & MRF $^{\mathbf{b}}$ & RSD (\%) $^{\mathbf{c}}$ \\
\hline 2-NT & 0.883 & 1.085 & 0.687 & 0.950 & 0.951 & 1.012 & 0.928 & 14.7 \\
3-NT & 0.678 & 0.925 & $d$ & 0.829 & 0.954 & 0.814 & 0.840 & 12.9 \\
4-NT & 0.840 & 1.052 & 0.839 & 0.869 & 0.898 & 0.713 & 0.868 & 12.6 \\
2,6-DNT & 0.823 & 0.702 & 0.823 & 0.753 & 0.567 & 0.794 & 0.743 & 13.2 \\
1,3-DNB & 0.182 & 0.152 & 0.197 & 0.190 & 0.197 & 0.167 & 0.181 & 10.2 \\
2,4-DNT & 0.631 & 0.593 & 0.648 & 0.549 & 0.473 & $\mathrm{e}$ & 0.579 & 12.2 \\
2-ADNT & 0.098 & 0.077 & 0.108 & 0.093 & 0.100 & $\mathrm{e}$ & 0.095 & 12.0 \\
4-ADNT & 0.092 & 0.067 & 0.087 & 0.078 & 0.072 & $\mathrm{e}$ & 0.079 & 13.3 \\
\hline
\end{tabular}

a Response Factor for each analyte relative to the internal standard;

${ }^{\mathrm{b}}$ Mean Response Factor (MRF);

c Relative Standard Deviation (RSD);

d Not measured due to co-elution of a septum contaminant;

${ }^{\mathrm{e}}$ Below the limit of quantification. 
Table 3. Results of spike-recovery experiments and published limits for solvent extraction of soil samples (CRL, MDL)

\begin{tabular}{rcccccc}
\hline \multicolumn{7}{c}{ Measured Concentrations $\left(\mathrm{mg} \mathrm{kg}^{-1}\right)$} \\
\hline & $\begin{array}{c}\text { Sample 1 } \\
0.4 \mathrm{mg} \mathrm{kg}^{-1}\end{array}$ & $\begin{array}{c}\text { Sample 2 } \\
0.2 \mathrm{mg} \mathrm{kg}^{-1}\end{array}$ & $\begin{array}{c}\text { Sample 3 } \\
0.1 \mathrm{mg} \mathrm{kg}^{-1}\end{array}$ & $\begin{array}{c}\text { Av. Rec. } \\
(\%)^{\mathrm{a}}\end{array}$ & $\begin{array}{c}\text { CRL }^{\mathbf{b}} \\
\mathrm{mg} \mathrm{kg}^{-1}\end{array}$ & $\begin{array}{c}\mathbf{M D L}^{\mathbf{c}} \\
\mathrm{mg} \mathrm{kg}^{-1}\end{array}$ \\
\hline 2-NT & 0.15 & 0.07 & 0.04 & 38 & 0.25 & 0.07 \\
3-NT & 0.15 & 0.07 & 0.04 & 38 & 0.25 & 0.07 \\
4-NT & 0.18 & 0.09 & 0.05 & 47 & 0.25 & 0.07 \\
2,6-DNT & 0.19 & 0.11 & 0.05 & 51 & 0.26 & 0.07 \\
1,3-DNB & 0.18 & 0.08 & 0.04 & 42 & 0.25 & 0.11 \\
2,4-DNT & 0.17 & 0.09 & 0.03 & 39 & 0.25 & 0.03 \\
2-ADNT & 0.12 & 0.06 & 0.02 & 27 & 0.25 & 0.03 \\
4-ADNT & 0.11 & 0.06 & 0.03 & 29 & 0.25 & - \\
\hline
\end{tabular}

${ }^{a}$ Average percentage of analyte recovery for the three spiked soil samples;

${ }^{\text {b }}$ Accepted Certified Reported Limits (CRL) according to the USATHAMA (1987) protocol;

${ }^{\mathrm{c}}$ Method Detection Limits (MDL)

\section{Qualitative Analysis of Soil Samples}

When real-world samples are not available, methods validation is commonly done by conducting spike-recovery studies. With these studies the matrix effects on the SPME method together with the overall recovery and analytical precision are investigated.

Previous attempts in extracting explosive residues from soil matrices were not very successful. The recommended procedure was to sonicate freshly prepared acetonitrile solutions containing the soil samples, then remove the organic solvent, add high salt content water and subsequently extract the analytes with SPME prior to chromatographic analysis (Darrach et al., 1998). Problems were encountered when using this procedure as the organic solvent must be preconcentrated if not eliminated from the samples destined for SPME. Even when a small amount of organic solvent is present in solution (e.g. $0.5 \% \mathrm{MeCN}$ :water), the analytes "prefer" to stay in solution instead of sorbing to the SPME fiber, making extraction impossible.

For the purpose of the present studies, a set of three soil samples were spiked with the analytes of interest, yielding target concentrations of 0.40 , 0.20 and $0.10 \mathrm{mg} \mathrm{kg}^{-1}$ dry soil and a different route of sample preparation was followed. The initial extraction was effectuated by sonicating the samples for $2 \mathrm{~h}$ after adding water with a high content in salt. The soil-slurry solutions were left to settle overnight in the dark. Once the supernatant was removed and the internal standard was added to it, the solution was analyzed following the SPME-GC/MS method for water samples. The results presented in Table 3 reveal low recovery for all target analytes and this was expected as water was used for extraction. Longer sonication times would most certainly lead to higher recoveries, and are indeed essential for detecting contaminants in real wold samples where analytes of interest are commonly bound tightly to soil particles. Nonetheless, at this stage, SPME detects analytes in levels of contamination below the Certified Reporting Limits (CRL) and Method Detection Limits (MDL) of solvent extraction for soil samples (Jenkins and Walsh, 1989; U.S. EPA Method 8330, 1994; Grant et al., 1989). This was achieved without even spiking the soils at SPME's Method Detection Limits. These experiments prove that SPME is not only an excellent screening method for explosive residues, but also detects contaminants in levels lower than the ones expected by the U.S.EPA Method 8330.

\section{CONCLUSIONS}

These findings are important for typical site characterization, where most samples submitted for analysis (up to $80 \%$ ) are blanks. The combination of SPME-GC/MS as a screening method will reduce the cost of analysis per sample and limit 
the production of hazardous laboratory waste (due to the traditional solvent extraction). These preliminary experiments revealed that the combination SPME-GC/MS is an excellent screening method for the trace analysis of explosive residues in soil detecting contaminants in levels lower than the ones reported in U.S. EPA Method 8330.

\section{REFERENCES}

Arthur, C.L., Potter, D.W., Buchholz, K.D., Motlagh, S. and Pawliszyn, J. (1992a), Solid-phase microextraction for the direct analysis of water: Theory and practice, Journal of Liquid Chromatography-Gas Chromatography, 10, 656-661.

Arthur, C.L., Killam, L.M., Buchholz, K.D., Pawliszyn, J. and Berg, J.R. (1992b), Automation and optimization of solid-phase microextraction, Analytical Chemistry, 64, 1960-1966.

Barshick, S.A. and Griest, W. (1998), Trace analysis of explosives in seawater using solid-phase microextraction and gas-chromatography/ion trap mass spectrometry, Analytical Chemistry, 70, 3015-3020.

Belardi, R.P. and Pawliszyn, J. (1989), The application of chemically modified fused silica fibers in the extraction of organics from water matrix samples and their rapid transfer to capillary columns, Water Pollution Research Journal of Canada, 24, 179-191

Berthe-Corti, L., Jacobi, H., Kleihauer, S. and Witte, I. (1998), Cytotoxicity and mutagenicity of (TNT) and hexogen contaminated soil in S. typhimurium and mammalian cells, Chemosphere, 2, 209-218.

Darrach, M.R., Chutjian, A. and Plett, G.A. (1998), Trace explosives signatures from World War II unexploded undersea ordnance, Environmental Science and Technology, 32, 1354-1358.

Eisert, R. and Levsen, K. (1996), Solid-phase microextraction coupled to gas chromatography: a new method for the analysis of organics in water, Journal of Chromatography A, 733, 143-157.

Frombert, A., Nilsson, T., Larsen, B.R., Montanarella, L., Facchetti, S. and Madsen, J.O. (1996), Analysis of chloro- and nitroanilines and -benzenes in soils by headspace solid-phase microextraction, Journal of Chromatography A, 746, 71-81.

Grant, L.C., Hewitt, A.D. and Jenkins, T.F. (1989), Comparisons of low concentration measurement capability estimates in trace analysis. Method detection limits and certified reporting limit, U.S. Army Cold Region Research and Engineering Laboratory, CRREL Report 89-202, Hanover, New Hampshire.

Hable, M., Stern, C., Asowata, C. and Williams, K. (1991), The determination of nitroaromatics and nitramines in ground and drinking water by wide-bore capillary gas chromatography, Journal of Chromatographic Science, 29, 131-135.

Jenkins, T.F. and Walsh, M.E. (1987), Development of an analytical method for explosive residues in soil, U.S. Army Cold Region Research and Engineering Laboratory, CRREL Report 87-7, Hanover, New Hampshire.

Jenkins, T.F. and Walsh, M.E. (1989), Development of an analytical method for the detection of explosive residues in soil, U.S. Army Cold Region Research and Engineering Laboratory, CRREL Report 89-9, Hanover, New Hampshire.

Reddy, G., Reddy, T.V., Choudhury H., Daniel, F.B. and Leach, G.J. (1997), Assessment of environmental hazards of 1,3,5-trinitrobenzene, Journal of Toxicology, Environment and Health, 5, 447-460.

Sarrion, M.N., Santos, F.J. and Galceran, M.T. (1998), Strategies for the Analysis of Chlorobenzenes in Soils Using Solid-Phase Microextraction Coupled with Gas-Chromatography-Ion Trap Mass Spectrometry, Journal of Chromatography A, 819, 197-209.

Tugulea, A.M., Sarna, L.P. and Webster, G.R.B. (1998), Solid-phase Microextraction (SPME) of the herbicide atrazine in aqueous soil suspensions, International Journal of Environmental Analytical Chemistry, 68, 137-153.

U.S. EPA Method 8000B (1996), Determinative chromatographic separations. U.S. Environmental Protection Agency, Office of Solid Waste and Emergency Response, Washington, D.C., SW-846 Revision 2.

U.S. EPA Method 8330 (1994), Nitroaromatics and nitramines by high performance liquid chromatography. U.S. Environmental Protection Agency, Office of Solid Waste and Emergency Response, Washington, D.C. SW-846 Revision 0. 
Walsh, M.E. (1990), Environmental transformation products of nitroaromatics and nitramines. Literature Review and recommendations for analytical development, U.S. Army Cold Region Research and Engineering Laboratory, CRREL Report 90-2, Hanover, New Hampshire.

Walsh, M.E. and Ranney, T.A. (1998), Determination of nitroaromatics, nitramines and nitrate ester explosives in water using SPE and GC-ECD, U.S. Army Cold Region Research and Engineering Laboratory, CRREL Report 98-2, Hanover, New Hampshire.

Walsh, M.E., Taylor, S. and Thorne, P.G. (1996), Development of an analytical method for white phosphorus (P4) in water and sediment using solid-phase microextraction, U.S. Army Cold Region Research and Engineering Laboratory, CRREL Report 96-16, Hanover, New Hampshire.

Zhang, Z. and Pawliszyn, J. (1993), Headspace solid-phase microextraction, Analytical Chemistry, 65, 1843-1852.

Zhang, Z., Yang, M.J. and Pawliszyn, J. (1994), Solid-phase microextraction, Analytical Chemistry, 66, 844A-853A. 